\title{
How Accurate is the Determination of Equilibrium Structures for van der Waals Complexes? The Dimer $\mathrm{N}_{2} \mathrm{O} \cdots \mathrm{CO}$ as an Example
}

\author{
Jean Demaison, ${ }^{1, a)}$ Natalja Vogt, ${ }^{1,2,}$ Yan Jin,,${ }^{3,4}$ Rizalina Tama Saragi,,${ }^{3}$ Marcos Juanes, ${ }^{3}$ Alberto \\ Lesarri $^{3}$
}

${ }^{1}$ Section of Chemical Information Systems, University of Ulm, Albert Einstein Allee 47, 89081 Ulm, Germany,

${ }^{2}$ Department of Chemistry, Lomonosov Moscow State University, Moscow, 119992 Moscow, Russian Federation,

${ }^{3}$ Departamento de Química Física y Química Inorgánica, Facultad de Ciencias - I.U. CINQUIMA, Universidad de Valladolid, Paseo de Belén, 7, 47011 Valladolid, Spain

${ }^{4}$ Permanent address: Department of Chemistry, School of Chemistry and Chemical Engineering, Chongqing University, Daxuecheng South Rd. 55, 401331, Chongqing, China

a) Author to whom correspondence should be addressed: jean.demaison@gmail.com 


\begin{abstract}
Plausible methods for accurate determination of equilibrium structures of intermolecular clusters have been assessed for the van der Waals dimer $\mathrm{N}_{2} \mathrm{O} \cdots \mathrm{CO}$. In order to assure a large initial dataset of rotational parameters we first measured the microwave spectra of the ${ }^{15} \mathrm{~N}_{2} \mathrm{O} \cdots{ }^{12} \mathrm{CO}$ and ${ }^{15} \mathrm{~N}_{2} \mathrm{O} \cdots{ }^{13} \mathrm{CO}$ isotopologues, expanding previous measurements. Then, an anharmonic force field was calculated ab initio and a semiexperimental equilibrium structure was determined. The dimer structure was also calculated at the coupled-cluster level of theory using very large basis sets with diffuse functions and counterpoise correction. It was found that the contributions of the diffuse functions and of the counterpoise correction are not additive and do not compensate each other although have almost the same value but opposite signs. The semiexperimental and ab initio structures were found in fair agreement, the equilibrium distance between the center of mass of both monomers being 3.825(13) $\AA$ and the intermolecular bond length $r\left(\mathrm{C}^{\cdots} \mathrm{O}\right)=3.300(9) \AA$. In this particular case, the mass-dependent method did not permit to determine reliable intermolecular parameters. The combination of experimental rotational constants and results of ab initio calculations thus proves very sensitive to examine the accuracy of structural determinations in intermolecular clusters, offering insight for other aggregates.
\end{abstract}




\section{INTRODUCTION}

Non-covalent interactions (NCIs) play a fundamental role in different fields in physics and chemistry, like catalysis, crystal packing or biology, and show a growing two-fold tendency: 1) to advance from fundamental aspects to the design of new compounds and supramolecular assemblies, ${ }^{1,2}$ and 2) to move from the conventional hydrogen bond ${ }^{3}$ to other bridge interactions involving many different atoms out of the first row. ${ }^{4,5}$ NCIs are much studied experimentally and theoretically, but only gasphase experiments ${ }^{6,7}$ reveal the properties of the isolated molecules and may be used for highresolution observations. Nevertheless, it is often difficult to estimate the accuracy of a molecular structure. This is particularly true in the case of intermolecular clusters because the only reliable way is to compare the results of different methods, e. g. high-level ab initio calculations and spectroscopic semiexperimental determinations. However, whereas ab initio optimizations are routine work (at least for small molecules), it is still difficult to obtain accurate experimental or semiexperimental structures for weakly-bound complexes because of the presence of large amplitude motions. For instance, Kraus et al. ${ }^{8}$ determined the semiexperimental structure of 16 complexes and found a root-mean-square error of $0.055 \AA$ for the bond lengths. This mediocre result will be confirmed by the present work, and it is definitely much worse that what can be achieved for semirigid molecules (about 0.001-0.002 $\AA$ ). ${ }^{9}$

For moderately-sized aggregates not perturbed by strong large-amplitude motions, rotational spectroscopy provides direct structural information through the moments of inertia, together with force-field parameters like the centrifugal distortion constants and electric or magnetic properties (i.e., nuclear quadrupole coupling parameters, etc.). ${ }^{10-12}$ These parameters permit to derive effective or substitution empirical structures ${ }^{13,14}$ but their precision is limited. Kisiel estimates that the accuracy for the valence angles is not better than $1^{\circ}$, and, for instance, the bond axis does not always coincide with the principal axis of the quadrupole tensor. ${ }^{15}$ On the other hand, ab initio methods can give good results ${ }^{16,17}$ but the convergence is very slow and requires very large basis sets. Furthermore, diffuse functions have a large effect and the basis set superposition error (BSSE) is far from negligible.

We will try in this article to estimate the accuracy achievable for the structure of a van der Waals complex using different methods combining experimental rotational data and ab initio calculations. We chose the dimer $\mathrm{N}_{2} \mathrm{O} \cdots \mathrm{CO}$ for three reasons: i) it has only five atoms (all first-row), simplifying calculations ii) it is known to behave as a semirigid molecule (the rovibrational spectrum can be analyzed with a conventional Hamiltonian), and iii) $\mathrm{CO}$ and $\mathrm{N}_{2} \mathrm{O}$ are two important greenhouse gases which may interact in the atmosphere.

The high-resolution infrared spectrum of $\mathrm{N}_{2} \mathrm{O} \cdots \mathrm{CO}$ was first measured by $\mathrm{Xu}$ and McKellar ${ }^{18}$ in the $\mathrm{CO}$ stretching region near $2150 \mathrm{~cm}^{-1}$. At about the same time, the infrared spectrum was measured in the region of the $v_{3} \mathrm{~N}_{2} \mathrm{O}$ monomer vibrational band near $2224 \mathrm{~cm}^{-1}$ by Qian and Howard. ${ }^{19}$ With the help of an intermolecular potential, they were able to determine an approximate 
structure for the T-shaped complex with the carbon atom pointing to $\mathrm{N}_{2} \mathrm{O}$, an interaction suggesting a tetrel bond. A little bit later, the rotational spectra of five isotopologues were measured by Ngari et al. ${ }^{20}$ who determined an approximate structure. The rotational constants of these five isotopologues should be more than sufficient to determine a structure. Unfortunately, several of the atoms (central nitrogen and both atoms of $\mathrm{CO}$ ) are quite close to the $b$-principal axis. Therefore, their isotopic substitution does not bring much new information. One way to palliate this difficulty, at least partially, is to use doubly substituted species. ${ }^{21}$ This was the argument for us to start the analysis of the ${ }^{15} \mathrm{~N}^{15} \mathrm{NO} \cdots \mathrm{CO}$ and ${ }^{15} \mathrm{~N}^{15} \mathrm{NO} \cdots{ }^{13} \mathrm{CO}$ isotopologues, previously unreported. For reasons of intensity the rotational measurements were limited to the global minimum. Another advantage of these isotopologues is that the absence of quadrupole hyperfine structure renders the analysis of the rotational spectra much easier. The structure of $\mathrm{N}_{2} \mathrm{O} \cdots \mathrm{CO}$ was first calculated at the MP2/6-311+G(d) level of theory by Venayagamoorthy and Ford. ${ }^{22}$ Quite recently, the infrared spectrum of a higherenergy isomer was detected by Barclay et al. ${ }^{23}$ This isomer has a similar skewed T-shaped structure but with the $\mathrm{CO}$ rotated ca. $180^{\circ}$, suggesting a $\mathrm{O} \cdots \mathrm{N}$ interaction.

\section{COMPUTATIONAL AND EXPERIMENTAL METHODS}

Most correlated-level ab initio electronic structure computations of the present study have been carried out at two levels: second-order Møller-Plesset perturbation theory ${ }^{24}$ (MP2) and coupled cluster (CC) theory with single and double excitation ${ }^{25}$ augmented by a perturbational estimate of the effects of connected triple excitations $[\mathrm{CCSD}(\mathrm{T})] \cdot{ }^{26}$ The Kohn-Sham density functional theory ${ }^{27}$ was also used with two functionals: B3LYP (Becke, 3-parameter, Lee-Yang-Parr ${ }^{28,29}$ hybrid) and the double-hybrid functional B2PLYP which combines exact Hartree-Fock exchange with a MP2-like correlation. ${ }^{30}$ This later functional is supposed to better take into account van der Waals forces. For a correct treatment of dispersion interactions the D3 model of Grimme ${ }^{31}$ employing the Becke-Johnson damping function (D3BJ) was used. ${ }^{32}$

The basis sets included correlation-consistent polarized $n$-tuple zeta cc-pVn $\mathrm{Z}^{33}$ functions with $n=\{\mathrm{D}, \mathrm{T}, \mathrm{Q}, 5,6\}$ abbreviated as $\mathrm{V} n \mathrm{Z}$ in the text. For intermolecular complexes, the inclusion of diffuse functions in the basis set is important. For this reason, the augmented VnZ (aug-cc-pVnZ, $\mathrm{AV} n \mathrm{Z}$ in short) basis sets ${ }^{34}$ were employed. The core-core and core-valence correlation effects on the computed equilibrium geometries ${ }^{35}$ were estimated using the correlation-consistent polarized weighted core-valence $n$-zeta (cc-pwCVnZ, wCVnZ in short, with $n=\mathrm{T}, \mathrm{Q})^{36}$ basis sets. In principle, for first-row atoms, it is sufficient to use the MP2 method to estimate this correction. ${ }^{37}$ In one case, the maug-cc-pVQZ basis set, mAVQZ in short, where all but the $s$ and $d$ diffuse functions are eliminated, was employed. ${ }^{38}$ The smaller Pople's $6-311+\mathrm{G}(3 \mathrm{df})$ basis set was also used..$^{39}$ The frozen 
core approximation (hereafter denoted as fc), i.e. keeping the 1s orbitals of the atoms doubly occupied during correlated-level calculations, was used in many calculations. Some geometry optimizations were also carried out by correlating all electrons (hereafter denoted as ae). The basis set superposition error (BSSE) was taken into account by the counterpoise (CP) correction. ${ }^{40}$ Finally, the Atom in Molecules (AIM) theory ${ }^{41,42}$ with its implementation in Gaussian by Cioslowski et al. ${ }^{43-49}$ was used. The calculations were performed at the B3LYP/6-311+G(3df,2pd) level of theory. The CCSD(T) calculations were performed with the MOLPRO $^{50,51}$ electronic structure program packages while most other calculations utilized the Gaussian09 and Gaussian16 programs. ${ }^{52}$

The microwave spectroscopy experiments used a pulsed-jet Fourier-transform microwave (FTMW) spectrometer based in the resonator design of Balle and Flygare, ${ }^{53}$ operating in the $8-18 \mathrm{GHz}$ frequency region. The spectrometer used a Fabry-Perot resonator $(\phi=33 \mathrm{~cm})$ with the coaxial orientation of the jet and resonator axis initiated by Grabow. ${ }^{54,55}$ Briefly, a supersonic jet was created by expansion of a gaseous mixture of ${ }^{15} \mathrm{~N}^{15} \mathrm{NO}$ and ${ }^{12} \mathrm{CO}$ or ${ }^{13} \mathrm{CO}$ (approximately $0.5 \%$ of each gas) into the expansion chamber. Neon was used as carrier gas, with stagnation pressures of ca. 1-3 bar. The electronic set-up in Figure S-I of Supplementary material is based in a CW microwave source, which is pulsed by a SPDT switch into the emitter or receiver channels. The excitation radiation is upconverted $30 \mathrm{MHz}$ with a single-side-band mixer and amplified up to $200 \mathrm{~mW}$. The radiation is then coupled into the cavity with dipole antennas, using a moving mirror to tune the cavity into resonance at each frequency. Typical excitation times are $1 \mu \mathrm{s}$. After the excitation cycle the timedomain emission signal is acquired in the time domain (typically $8 \mathrm{k}$ points or ca. $400 \mu \mathrm{s}$ ), using lownoise amplification and down-conversion to the $30 \mathrm{MHz}$ region with an image rejection mixer. The digitizer uses a $200 \mathrm{MHz}$ integrated oscilloscope. A Fourier transform finally produces the frequency domain spectrum. The microwave resonator has an effective bandwidth of ca. $1 \mathrm{MHz}$, so the cavity is retuned mechanically for frequency scanning under computer control. Most of the electronic setup, in particular the digitizer, signal control and motor movement, is contained in a PXI chassis. All spectrometer signals are referred to a rubidium frequency standard, and the uncertainty of the frequency measurements is taken as $5 \mathrm{kHz}$. Because of the coaxial orientation of the jet and resonator axis the final transition frequencies are split into Doppler doublets, so the resonance frequencies are taken as the average frequency of the Doppler components. For each transition frequency the experiment is typically averaged $10^{2}-10^{4}$ times with $1-5$ excitations per gas pulse. The experimental repetition rates are normally 5 or $10 \mathrm{kHz}$. The spectrometer is computer-controlled using Grabow's FTMW++ control software. 


\section{RESULTS}

\section{Ab initio and DFT calculations}

\subsection{AIM analysis}

The AIM results for the global minimum are given in Table I. The bond critical point density ( $\rho$ ) is a measure of the amount of electron density shared between the two bonded atoms and is roughly proportional to the bond length. The bond ellipticity $(\varepsilon)$ provides a measure of the extent to which the charge is preferentially accumulated at a given angle in a plane perpendicular to the bond path and, for this reason, is a measure of the $\pi$-character of the bond. ${ }^{41,42}$ Furthermore, the Laplacian $(\nabla)$ of the electron density is also given, showing where the field is locally concentrated $(\nabla<0)$ or depleted $(\nabla$ $>0$ ). When $\nabla<0$, the concentration of charges is between the atoms forming the bonds, as it is typical for covalent bonds. On the other hand, when $\nabla>0$ the charges are away from the internuclear region. Our high-level ab initio calculations and the AIM results on the resulting electron density confirmed the initial presumption that the structure of the monomers $\mathrm{CO}$ and $\mathrm{N}_{2} \mathrm{O}$ is not much affected by the complexation.

There are two possibilities for the intermolecular bond, either $\mathrm{C} \cdots \mathrm{N}$ (central) or C $\cdots$ O. Both bonds have about the same length, but the bond critical point density clearly indicates that the bond is between $\mathrm{C}$ and $\mathrm{O}$. It is also worth noting that the Laplacian $\nabla$ is positive confirming that the bonding is not covalent but van der Waals. Furthermore, the change of $\varepsilon$ upon complexation confirms the redistribution of the electronic charge. To explain the formation of this intermolecular bond, the charge of the atoms was calculated using two different methods: Natural Bond Orbital ${ }^{56}$ (NBO) and Charge Model $5^{57}$ (CM5), the later method having the reputation to be more accurate. In all cases, the carbon atom and the central nitrogen atoms are positive whereas the remaining atoms are negative, see Table II. Thus, the bond is between the electropositive $\mathrm{C}$ of $\mathrm{CO}$ and the electronegative $\mathrm{O}$ of $\mathrm{N}_{2} \mathrm{O}$. Note, however, that the bond is not only due to electrostatic attraction as shown by the existence of a non-zero bond critical point density between the atoms $\mathrm{C}$ and $\mathrm{O} 2\left(\rho=3.68 \cdot 10^{-3}\right)$. It is also worth noting that the charges on the atoms do not vary much upon complexation.

\subsection{Calculation of the anharmonic force field}

Kraus et al. ${ }^{8}$ have shown that the CP-B2PLYP-D3BJ/AVTZ level of theory permits to determine the semiexperimental structure of a complex with a bond length RMS error of $0.055 \AA$. This accuracy estimation is confirmed by the work of Obenchain et al. on the complex $\left(\mathrm{CH}_{3}\right)_{2} \mathrm{~S} \cdots \mathrm{SO}_{2}{ }^{58}$ This method was applied for $\mathrm{N}_{2} \mathrm{O} \cdots \mathrm{CO}$ using three different basis sets: $6-311+\mathrm{G}(3 \mathrm{df})$, AVTZ and mAVQZ. As 
further check, the CP-B3LYP-D3BJ/AVTZ level of theory was also employed, since the latter method has been applied successfully to thousands of different systems, including dimers. ${ }^{8,59-61}$ The results are given in Table III (CP-MP2/6-311+G(d) results of Ref. 22 are also included). Note that the $r(\mathrm{C} \cdots \mathrm{O})$ bond length is quite sensitive to the level of theory. The complete structures are given in Table S-I of the Supplementary material.

Concerning the harmonic force field, the theoretical quartic centrifugal distortion constants are in good agreement and close to the experimental values, see Table III. For instance, the experimental value of $D_{J K}$ is only $4 \%$ smaller than the computed value [B2PLYP-D3/6-311+G(3df)]. In addition, the experimental ground state inertial defect, $\Delta_{0}=1.935 \mathrm{u} \AA^{2}$ is close to the computed value, $1.982 \mathrm{u} \AA^{2}$. Furthermore, the harmonic vibrational frequencies from all calculations are close to each other and are also in good agreement with experimental values. From the comparison of the computed harmonic frequencies, the largest range is found for the CO stretch $\left(12.7 \mathrm{~cm}^{-1}\right)$. It may be concluded that the harmonic force field is likely to be reliable and not much dependent on the basis set. For this reason, the centrifugal distortion constant $D_{K}$ which could not be determined from the experimental spectra was fixed at the computed value (see Section 2).

Looking at the anharmonic vibrational frequencies, the situation is less favorable. Only two experimental vibrational frequencies are known. The experimental $\mathrm{N}_{2} \mathrm{O} \operatorname{stretch}^{19}$ at $2226.7 \mathrm{~cm}^{-1}$ is only $13.8 \mathrm{~cm}^{-1}$ larger than the computed value and the $\mathrm{CO}$ stretch ${ }^{18}$ at $2147.3 \mathrm{~cm}^{-1}$ is $1.9 \mathrm{~cm}^{-1}$ larger than the computed value [B2PLYP-D3/6-311+G(3df) in both cases], see also Table III. From the comparison of the computed harmonic frequencies, the largest range is found for the $\mathrm{CO}$ stretch, 12.7 $\mathrm{cm}^{-1}$, but there is obviously a problem with the low-frequency intermolecular vibrations which have an anharmonic correction much too large. This is particularly true for the $\mathrm{C}^{\cdots} \mathrm{O}$ stretch (the computed anharmonic contribution is $41.6 \mathrm{~cm}^{-1}$ whereas the harmonic frequency is $24.2 \mathrm{~cm}^{-1}$, CP-B2PLYP/6$311+\mathrm{G}(3 \mathrm{df})$ values, see Table III). It may be explained by the fact that the minimum of the potential hypersurface is very flat. Nevertheless, the different levels of theory give consistent rovibrational corrections which permit to derive a semiexperimental equilibrium inertial defect close to zero (see Section 3.1).

\subsection{Ab initio Born-Oppenheimer structure}

The CCSD(T)_ae/wCVQZ level of theory is known to give an accurate structure for a semirigid molecule, the mean error being only $0.002 \AA^{9}{ }^{9}$ However, this is no more true for a van der Waals complex where a larger basis set is required, diffuse functions have to be included and the counterpoise correction has to be taken into account. In such a case, use is made of a composite scheme in which the various contributions are evaluated separately at the highest possible level and 
summed up, which is suitable to account in an efficient manner for basis-set truncation as well as higher excitation and core-correlation effects. ${ }^{62}$

\subsubsection{Core correlation}

To reduce the computational effort, it is common to use the fc approximation, in which only the valence electrons are correlated. Then, the core-core and core-valence correlation corrections are calculated as the difference between the values from ae and fc calculations. It is well established that the $\operatorname{CCSD}(\mathrm{T}) / \mathrm{wCVTZ}$ level of theory recovers only about three quarters of the correction. ${ }^{35}$ For this reason, it is commonly calculated at the $\operatorname{CCSD}(\mathrm{T}) / \mathrm{wCVQZ}$ level. However, in some cases, the much cheaper MP2/wCVTZ method is used. It usually gives good results because there is a compensation of errors, the wCVTZ basis set underestimating the value while the MP2 method overestimates it. The two methods are compared in Table IV. While there is a good agreement, as expected, for the geometrical parameters of the monomers, the MP2/wCVTZ method hugely overestimates the correction of the intermolecular bond length. For the intermolecular bond $\mathrm{C} \cdots \mathrm{O} 2$, the core correction is $0.0055 \AA$ at the MP2/wCVTZ level of theory but only $0.0020 \AA$ at the CCSD(T)/wCVQZ one, whereas an extrapolation to infinite basis set gives $0.0017 \AA$. Thus, the MP2/wCVTZ method cannot be used for a complex.

It remains to check whether the wCVQZ is large enough to recover all the correlation. For this goal, the $\operatorname{CCSD}(\mathrm{T}) / \mathrm{wCVTZ}$ and $\operatorname{CCSD}(\mathrm{T}) / \mathrm{wCVQZ}$ were extrapolated using the following equation

$$
\Delta r(C B S)=\frac{n^{3} r(n)-(n-1)^{3} r(n-1)}{n^{3}-(n-1)^{3}}-r(n-1)
$$

where $n$ indicates the cardinal number of the larger basis set. This extrapolation formula is known to have an accuracy of about $0.001 \AA$ when only first-row atoms are involved, at least for covalent bonds. ${ }^{63}$ The conclusion is that the infinite basis set values are very close to the wCVQZ ones.

\subsubsection{Contribution of diffuse functions}

The next correction to be considered is the contribution of the diffuse functions. It is known that this correction can be estimated at the MP2 level but, what is still more interesting, it decreases rapidly with the size of the basis set. It is confirmed by inspection of Table V, see also Table S-II of the Supplementary Material for detailed results. Furthermore, when the basis set is large enough (AVQZ or larger), the correction is important only for the intermolecular bond lengths $\mathrm{C} \cdots \mathrm{O}$ and $\mathrm{C} \cdots \mathrm{N}$. At the AVTZ level, the effect of the diffuse functions is to decrease the $\mathrm{C} \cdots \mathrm{O}$ bond length by as much as $0.042 \AA$ whereas, with the AV6Z basis set, the decrease is a small as $0.008 \AA$. The comparison between AV5Z and AV6Z shows that convergence is almost achieved. However, the AV6Z values of the intermolecular bond lengths $r_{\mathrm{e}}\left(\mathrm{C}^{\cdots} \mathrm{N} 2\right)$ and $r_{\mathrm{e}}\left(\mathrm{C}^{\cdots} \mathrm{O}\right)$ are still smaller by $0.008 \AA$ than the $\mathrm{V} 6 \mathrm{Z}$ values. Indeed, extrapolation at infinite basis size using the Eqn. (1) shows that the $\mathrm{VnZ}$ and $\mathrm{AVnZ}$ 
basis sets converge towards the same limit with the exception of the intermolecular bond lengths $\mathrm{C} \cdots \mathrm{N}$ and $\mathrm{C} \cdots \mathrm{O}$ for which the difference is still not negligible, the extrapolated values MP2/VœZ being too large. However, it is important to remember that the extrapolation ends to overestimate the basis-set truncation error and that the convergence of the MP2/VnZ values is not smooth. Finally, a perhaps more rational explanation is that diffuse functions are required for intermolecular bonds. Indeed, the comparison between the mAVQZ and the AVQZ results is also interesting. It shows that a full set of diffuse functions is required: for instance, at the CP-B2PLYP-D3 level, the $\mathrm{C} \cdots \mathrm{O}$ bond length is $3.3444 \AA$ with the mAVQZ basis set and $3.3285 \AA$ with the AVQZ basis set, see Table S-I of the Supplementary Material.

\subsubsection{Counterpoise correction}

Finally, the effect of the counterpoise correction is illustrated in Table VI. It was first calculated at the MP2 level of theory. It is large for small basis sets but decreases with the size of the basis set. Furthermore, it does not affect the structure of the monomers. Actually, as for the effect of diffuse functions, it is important only for the intermolecular bond. With the AVQZ basis set, the counterpoise correction increases the $\mathrm{C} \cdots \mathrm{O}$ bond length by $0.023 \AA$ but with the AV6Z basis set, the increase is already as small as $0.005 \AA$. The extrapolation to infinite basis size with Eq. (1) confirms that both structures, with or without counterpoise correction, converge towards the same limit, albeit slowly. Finally, it is worth noting the effects of the diffuse functions and of the counterpoise correction seem to partly compensate each other. This correction is further discussed in the next section 1.3.4.

\subsubsection{Structure optimization}

Different methods were used. First, the structure was optimized at the CCSD(T)_ae/wCVQZ level of theory,

$$
r_{\mathrm{e}}(\mathrm{Q})=r_{\mathrm{e}}\left(\mathrm{CCSD}(\mathrm{T}) \_\mathrm{ae} / \mathrm{wCVQZ}\right) .
$$

For a semi-rigid molecule with only first-row atoms, a mean-error of about $0.001 \AA$ is achievable with this method. ${ }^{64}$ The results are given in Table VII. Before going further, it is important to compare the structure of $\mathrm{N}_{2} \mathrm{O}$ and $\mathrm{CO}$ in the complex to the structure of the monomers computed at the same level of theory. For the complex (in $\AA$ ): $r(\mathrm{CO})=1.1289 ; r(\mathrm{NN})=1.1262 ; r(\mathrm{NO})=1.1847$ whereas for the monomers the results are: $r(\mathrm{CO})=1.1286$ (semiexperimental value: $\left.1.1284^{9}\right) ; r(\mathrm{NN})=$ $1.1267 ; r(\mathrm{NO})=1.1848\left(\right.$ experimental values: $\left.r(\mathrm{NN})=1.1273 ; r(\mathrm{NO})=1.1851^{65}\right)$. This confirms that the structure of the monomers is very slightly affected by the complexation.

The problem is that, for the van der Waals bond, the convergence is obviously not achieved. For this reason, the effect of the basis set extension was estimated at the MP2 level.

$$
\begin{gathered}
r \mathrm{e}(5)=r_{\mathrm{e}}\left(\mathrm{CCSD}(\mathrm{T}) \_\mathrm{ae} / \mathrm{wCVQZ}\right)+\Delta r\left[\mathrm{MP} 2 \_\mathrm{ae} /(\mathrm{wCV} 5 \mathrm{Z}-\mathrm{wCVQZ})\right] \\
r_{\mathrm{e}}(6)=r \mathrm{e}\left(\mathrm{CCSD}(\mathrm{T}) \_\mathrm{ae} / \mathrm{wCVQZ}\right)+\Delta r\left[\mathrm{MP} 2 \_\mathrm{ae} /(\mathrm{wCV} 5 \mathrm{Z}-\mathrm{wCVQZ})\right]+\Delta r\left[\mathrm{MP} 2 \_\mathrm{fc} /(\mathrm{V} 6 \mathrm{Z}-\right.
\end{gathered}
$$

V5Z)]. 
The results are also given in Table VII. It is not obvious that convergence is achieved at the V6Z level. To check this point, Eqn. (1) was used to extrapolate the $r_{\mathrm{e}}(5)$ and $r_{\mathrm{e}}(6)$ results, although this formula is not expected to be accurate in the present case because the convergence is not smooth. The extrapolated values are called $r_{\mathrm{e}}(\infty)$. They are close to $r_{\mathrm{e}}(6)$ and the small differences may be used to estimate the uncertainty. It is probable that, at this level, the effect of diffuse functions and counterpoise correction are almost negligible. Indeed, at the MP2/AV6Z level, the effect of the diffuse functions is to decrease the $\mathrm{C}^{\cdots} \mathrm{O}$ bond length by $0.008 \AA$ whereas the counterpoise correction increases it by $0.006 \AA$ (see Tables V and VI). In other words, the $\mathrm{C} \cdots \mathrm{O}$ bond length might be 0.001 $\AA$ too long. It is also interesting to have a look at the other parameters. The intermolecular bond angles do not vary much from ${ }^{\mathrm{NCVQZ}}$ to $\mathrm{WCV} \infty \mathrm{Z}$, the range being smaller than $0.35^{\circ}$. With the increase of the basis set, the intramolecular bond lengths sightly decrease. It is indeed known that, at the $\operatorname{CCSD}(\mathrm{T}) / \mathrm{wCVQZ}$ level of theory, the bond lengths are slightly too long by about $0.0004(6) \AA .{ }^{66}$ This good result is mainly due to a compensation of errors because the introduction of connected quadruples (CCSDTQ) increases the bond length by 0.001-0.002 $\AA$ whereas the basis set extension from quadruple zeta to sextuple zeta shortens it by about the same amount. In other words, extension of the basis set beyond wCVQZ does not improve the accuracy of the intramolecular bond lengths and may even deteriorate it.

To check these results, the structure was also calculated with this equation

$$
r_{\mathrm{e}}(\mathrm{II})=r_{\mathrm{e}}\left(\mathrm{CCSD}(\mathrm{T}) \_\mathrm{fc} / \mathrm{AVQZ}\right)+\Delta r(\text { core })+\Delta r(\mathrm{CBS})+\Delta r(\mathrm{CP})
$$

with $\Delta r(\mathrm{CBS})$ computed from Eqn. (1) with the results MP2_fc/AV $n \mathrm{Z}$ with $n=\mathrm{Q}, 5$ (see Table S-III) and

$$
\Delta r(\text { core })=\Delta r\left(\operatorname{CCSD}(\mathrm{T}) \_\mathrm{ae} / \mathrm{wCVQZ}-\mathrm{CCSD}(\mathrm{T}) \_\mathrm{fc} / \mathrm{wCVQZ}\right)
$$

The results, given in Table VII, are almost identical to either $r_{\mathrm{e}}(6)$ or $r_{\mathrm{e}}(\infty)$. Actually, the $\mathrm{C} \cdots \mathrm{O}$ bond length is about $0.002 \AA$ shorter than the $r_{\mathrm{e}}(\infty)$ value, as predicted although it seems to indicate that the counterpoise correction is almost negligible.

For the sake of completeness, the structure was also calculated starting from the $\operatorname{CCSD}(\mathrm{T}) \_f c / V Q Z$ values with the equation

$$
r_{\mathrm{e}}(\mathrm{III})=r_{\mathrm{e}}\left(\mathrm{CCSD}(\mathrm{T}) \_\mathrm{fc} / \mathrm{VQZ}\right)+\Delta r(\text { core })+\Delta r(\mathrm{CBS})
$$

with $\Delta r$ (core) from Eqn. (6) and $\Delta r(\mathrm{CBS})$ computed from Eqn. (1) with the results MP2_fc/VnZ with $n=\mathrm{Q}, 5$. The results of Eqn. (7), which neglects both the diffuse functions and the counterpoise correction, are not in agreement with the results of Eqns. $(3,4,6)$, the $\mathrm{C} \cdots \mathrm{O}$ bond length being much shorter: $3.294 \AA$ instead of $3.307 \AA$. This is surprising because the absence of diffuse functions should lead to an increase of the $\mathrm{C} \cdots \mathrm{O}$ bond length, see Table V. For this reason, as a further check, the structure was also calculated with the following equation

$$
r_{\mathrm{e}}(\mathrm{IV} n)=r_{\mathrm{e}}\left(\mathrm{CP}-\mathrm{CCSD}(\mathrm{T}) \_\mathrm{fc} / \mathrm{AV} n \mathrm{Z}\right)+\Delta r(\text { core })
$$


where $n=\mathrm{T}, \mathrm{Q}, 5$ and $\Delta r$ (core) from Eqn. (6). For the results, see Table VII. As the convergence is not yet achieved at the AV5Z level (for instance, the $\mathrm{C}^{\cdots} \mathrm{O}$ bond length decreases of $0.028 \AA$ when going from AVTZ to AVQZ and of further $0.009 \AA$ when going from AVQZ to AV5Z), the correction $5 \rightarrow 6$ was estimated at the MP2 level. Finally, the $r_{\mathrm{e}}(\mathrm{IV} n)$ were extrapolated to infinite basis set using Eqn. (1) and starting from $n$ equal either Q, 5 or 6 , see Table VII. The $\mathrm{C} \cdots \mathrm{O}$ bond length decreases monotonously with the increase of the basis set. The extrapolations ( $\rightarrow \mathrm{Q}$ and $\mathrm{Q} \rightarrow 5$ ) give consistent values: $3.295 \AA$, in perfect agreement with $r(\mathrm{III})$. On the other hand, the extrapolation $5 \rightarrow 6$ gives a smaller value: $3.290 \AA$. It is difficult to estimate which extrapolation gives the most accurate value: in principle, it should be the $5 \rightarrow 6$ one but, as the extension of the basis set from AV5Z to AV6Z was only calculated at the MP2 level, it could be less accurate. Actually, this difference might be used to estimate the accuracy.

Finally, it is useful to make a comparison with the previous results. A surprising result is that the values of the bond angles depend on the method. For instance, the value of the $\angle(\mathrm{OCO})$ bond angle is about $145^{\circ}$ at the CP-CCSD(T) level whereas it is $154^{\circ}$ at the MP2 level, see Tables VI and VII (the CP-B2PLYP-D3 method with the AVQZ basis set is only marginally better yielding $\angle$ (OCO) $=148.8^{\circ}$ ). A similar behavior was previously observed for the torsional angles ${ }^{67}$ where the MP2 method fails to deliver accurate values. It was explained by the fact that it requires much less energy to modify a torsional angle than a bond angle. In the case of $\mathrm{N}_{2} \mathrm{O} \cdots \mathrm{CO}$, the force constant of the intermolecular bond angles is as small as (or even smaller than) that of the torsional angles.

As a conclusion, a satisfactory agreement was found for all parameters except for the intermolecular bond length where differences as large as $0.010 \AA$ are observed. In particular, the $r_{\mathrm{e}}(\mathrm{IV} 5)$ and $r_{\mathrm{e}}(\mathrm{IV} \infty)$ bond lengths are shorter by $0.013 \AA$ compared to either $r_{\mathrm{e}}(\infty)$ or $r_{\mathrm{e}}(\mathrm{II})$. It is known that the counterpoise correction is only an approximation and that the correction may be too large. ${ }^{68}$ There is another possible explanation. It is well established that the compound method which assumes that the different small corrections (diffuse functions, core correlation, extension of the basis set, ...) are additive (see Eqs. (3) to (5)) is accurate for rigid molecules. This is no more obvious when the counterpoise correction is involved. To check this point, the CP-MP2/AVQZ structure was calculated both directly and using Eqn. (8)

$$
\begin{aligned}
r_{\mathrm{e}}(\mathrm{CP}-\mathrm{MP} 2 / \mathrm{AVQZ}) & \approx r_{\mathrm{e}}(\mathrm{MP} 2 / \mathrm{VQZ})+\Delta r(\mathrm{CP}-\mathrm{MP} 2 / \mathrm{VQZ}-\mathrm{MP} 2 / \mathrm{VQZ}) \\
& +\Delta r(\mathrm{MP} 2 / \mathrm{AVQZ}-\mathrm{MP} 2 / \mathrm{VQZ})
\end{aligned}
$$

The complete results are given in Table S-IV of the Supplementary Material. As expected, this equation gives accurate results for the bond lengths of the monomers but it fails for the intermolecular parameters. For instance, the exact CP-MP2/AVQZ value of $r_{\mathrm{e}}(\mathrm{C} \cdots \mathrm{O})$ is $3.330 \AA$ whereas Eqn. (9) gives $3.341 \AA$. The intermolecular bond angles are also significantly affected. In other words, although the contributions of the diffuse functions and of the counterpoise correction have almost the 
same value but opposite signs, they are not additive and do not compensate each other. For this reason, the $r_{\mathrm{e}}(\mathrm{IV})$ values are probably more accurate.

\section{Analysis of the rotational spectra}

When the ground state rotational spectrum of the parent species was analyzed, it was possible to determine all quartic centrifugal distortion constants using the Watson's semi-rigid Hamiltonian. However, the experimental value of $D_{K}=282.097(53) \mathrm{kHz}$, has a sign opposite to the calculated values whereas the agreement is quite good for the other four constants, see Table III. This experimental value of $D_{K}$ leads to a negative $T_{a}$ which, furthermore, has the correct order of magnitude. Nevertheless, there is obviously a compatibility problem. For this reason, a new fit of the rotational spectrum was performed. It appeared that one transition, $3_{22}-4_{31}$ at $17657 \mathrm{MHz}$ was an influential point, the different statistical diagnostics indicating that $D_{K}$ was determined by this transition alone $\left(h_{i i}=1\right.$ and DFBETAS $=23819$ instead of being smaller than 40). Indeed, a new fit without this transition did not permit to determine $D_{K}$.

For this reason, the spectra of all isotopologues were fitted with the help of the computed centrifugal distortion constant $D_{K}$ (CP-B2PLYP-D3/mAVQZ value). For the species with two ${ }^{14} \mathrm{~N}$ nitrogen atoms, $D_{K}$ was fixed at the computed value and, for the other species, the method of predicate observations $^{69-71}$ was used. It combines the experimental rotational frequencies with quartic centrifugal distortion constants derived from the harmonic force field. The uncertainty used for the weighting the predicates was $10 \%$ of their value. The results of the fits are given in Table S-V of the Supplementary Material. The newly measured transitions of ${ }^{15} \mathrm{~N}_{2} \mathrm{O} \cdots{ }^{12} \mathrm{CO}$ and ${ }^{15} \mathrm{~N}_{2} \mathrm{O} \cdots{ }^{13} \mathrm{CO}$ are given in Tables S-VI and S-VII of the Supplementary Material. Unfortunately, it was not possible to assign any transition of the $\mathrm{NN}^{18} \mathrm{O}$ species. It is easily explained by the low abundance of ${ }^{18} \mathrm{O}(0.2$ $\%)$ and the small dipole moment of the complex which is smaller than 0.3 D (B2PLYP/AVQZ and $\operatorname{CCSD}(\mathrm{T}) / \mathrm{AVQZ}$ results).

\section{Experimental structures}

\subsection{Determination of the semiexperimental rotational constants}

The semiexperimental equilibrium rotational constants, $X_{\mathrm{e}}(X=A, B, C)$, are obtained from the ground state rotational constants, $X_{0}$, using the following expression

$$
X_{\mathrm{e}}=X_{0}+\Delta X_{\mathrm{cd}}+\Delta X_{\mathrm{g}}+\Delta X_{\mathrm{vib}}
$$

where $\Delta X_{\text {vib }}$ is by far the largest correction and will be discussed in the next section. $\Delta X_{\mathrm{g}}$ is a small correction due to the fact that atomic masses are used to calculate the structure although the center of mass of the electrons of a bonded atom often does not coincide with the position of its nucleus because 
the distribution of the electronic clouds around the atoms in a molecule is non-spherical. ${ }^{72}$ For this reason, the rotational constants have to be corrected for a small electronic effect. The corrected values of the rotational constants are given by the relation

$$
X_{\mathrm{corr}}=\frac{X_{\mathrm{exp}}}{1+\frac{m}{M_{\mathrm{P}}} g_{\mathrm{xx}}}
$$

where $g_{\mathrm{xx}}$ is expressed in nuclear magnetons, $m$ is the electron mass and $M_{\mathrm{P}}$ the proton mass. Experimental values of $g_{x x}$ are not known, so approximate values were calculated at the B3LYP/6$311+\mathrm{G}(3 \mathrm{df})$ level of theory. The values are: $g_{a a}=-0.0843 ; g_{b b}=-0.0091$; and $g_{c c}=-0.0843$, leading to the following corrections (in MHz): $\Delta A_{\mathrm{g}}=0.579 ; \Delta B_{\mathrm{g}}=0.010$; and $\Delta C_{\mathrm{g}}=0.017 . \Delta X_{\mathrm{cd}}$ is a small centrifugal distortion correction because the experimental rotational constants are slightly different from the rigid rotor constants. It is often neglected because of its smallness but, in the particular case of a floppy molecule, it is larger and it is better to take it into account. For instance, for the parent species (in MHz): $\Delta A_{\mathrm{cd}}$ $=-0.370 ; \Delta B_{\mathrm{cd}}=-0.001 ;$ and $\Delta C_{\mathrm{cd}}=0.187$. After the centrifugal and electronic corrections, the ground state inertial defect is $\Delta_{0}=1.935 \mathrm{u} \AA^{2}$. The lowest-frequency vibration is the in-plane C $\cdots \mathrm{O}$ stretch. Assuming that this vibration is well-isolated (which is disputable), $\Delta_{0}$ permits to estimate the vibrational frequency: $v=16.863 / \Delta_{0}$, it gives $v=33 \mathrm{~cm}^{-1}$.

The theoretical rovibrational corrections deduced from the CP-B2PLYP-D3/6-311+G(3df) anharmonic force field (quadratic and cubic terms) were combined with the ground state rigid rotor constants to yield the semiexperimental equilibrium rotational constants. The values for the parent species are given in Table III and those of the isotopologues in Table S-VIII of the Supplementary Material. It is worth noting that with these semiexperimental constants, the equilibrium inertial defect, $\Delta_{0}=-0.006 \mathrm{u} \AA^{2}$, is close to zero indicating that the rovibrational corrections might be accurate. It is also interesting to remark that the isotopic variation of these corrections is small, less than $6 \%$ for $\Delta A_{\text {vib }}$ and less than $10 \%$ for $\Delta B_{\text {vib }}$ and $\Delta C_{\text {vib. }}$

\subsection{Pseudodiatomic approximation}

Assuming that the monomer geometries are unchanged upon complexation (which was shown to be a good approximation in Section 1.3.4) it is possible to express the moments of inertia of the complex as a function of the moments of inertia of the monomers in addition to three parameters: the distance $R_{\mathrm{cm}}$ between the center of mass of the two monomers $\left(\mathrm{cm}_{1}\right.$ and $\left.\mathrm{cm}_{2}\right)$ and two angles, $\theta_{1}=$ $\angle\left(\mathrm{cm}_{1} \ldots \mathrm{cm}_{2} \ldots \mathrm{O} 2\right)$ and $\theta_{2}=\angle\left(\mathrm{cm}_{2} \ldots \mathrm{cm}_{1} \ldots \mathrm{C}\right) .{ }^{19}$ From the moment of inertia $I_{c}$, it is possible to deduce $R_{\mathrm{cm}}=3.3106(6) \AA$ with a small range of $0.0006 \AA$ for seven values, indicating that the different $C_{\mathrm{e}}$ values are consistent. Then, the fit of $I_{a}$ and $I_{b}$ as data permits to determine $\theta_{1}$ and $\theta_{2}$. Only using either $I_{a}$ or $I_{b}$ gives almost identical results. Analysis of the residuals indicates that there is a small systematic error of about $0.95 \mathrm{MHz}$ for $A_{\mathrm{e}}$ and $11 \mathrm{kHz}$ for $B$ e. Furthermore, the rotational 
constants of the ${ }^{15} \mathrm{~N}^{14} \mathrm{NO}$ species are outliers. It may be partly explained by the fact that this ${ }^{15} \mathrm{~N}$ atom is far from the center of mass. Comparison of these results with the ab initio results of section 1.3.4, see Table VII, shows a large difference for the $\theta_{1}$ angle which is $83.75(5)^{\circ}$ compared to about $80^{\circ}$ for the different ab initio results. The value of the $\theta_{2}$ angle at $9.38(1)^{\circ}$ is in better agreement with the ab initio values. The $R_{\mathrm{cm}}$ value at $3.81 \AA$ is also shorter than the ab initio values which are about $3.82 \AA$. These inconsistencies may be explained in different ways. It may be due to the fact that the geometries of $\mathrm{N}_{2} \mathrm{O}$ and $\mathrm{CO}$ are frozen to the monomer values. A sensitivity analysis indicates that the uncertainty on the moments of inertia of $\mathrm{N}_{2} \mathrm{O}$ and $\mathrm{CO}$ plays a negligible role. On the other hand, as expected, $R_{\mathrm{cm}}$ is quite sensitive to the value of $C_{\mathrm{e}}$. The problem is that the $\theta_{1}$ angle is also the most sensitive to the constant $C_{\mathrm{e}}$. The derived value of $R_{\mathrm{cm}}$ seeming to be too small indicates that $C_{\mathrm{e}}$ is too large $\left(\partial R_{\mathrm{cm}} / \partial C_{\mathrm{e}}\right.$ $\left.=-0.0013 \AA \mathrm{MHz}^{-1}\right)$ but $\theta_{1}$ being too large leads to the conclusion that $C_{\mathrm{e}}$ is too small $\left(\partial \theta_{1} / \partial C_{\mathrm{e}}=-0.43\right.$ degree $\mathrm{MHz}^{-1}$ ). These two conclusions are obviously not compatible. It confirms the limited accuracy of this method. However, it cannot be excluded that the rovibrational corrections are affected by a large systematic error.

\subsection{Semiexperimental structure}

The structure was fitted to the semiexperimental moments of inertia. To improve the quality of the fit, predicate observations were used for the geometrical parameters of both monomers. The chosen values are the $\operatorname{CCSD}(\mathrm{T}) \_$ae/wCVQZ results, being likely close to the true equilibrium values due to a well-documented compensation of errors, namely, due to the introduction of connected quadruples (CCSDTQ) increasing the bond length by 0.001-0.002 $\AA$ and the basis set extension from quadruple zeta to sextuple zeta shortening it by about the same amount. ${ }^{73}$ In this particular case, the choice of the molecular parameters to be fitted has some importance. We may choose to attach the $\mathrm{C}$ atom either to one nitrogen atom or to the end oxygen atom of $\mathrm{N}_{2} \mathrm{O}$. As the carbon atom is closest to the central nitrogen atom, it would seem reasonable to use the $\mathrm{C} \cdots \mathrm{N} 2$ bond length as parameter. Unfortunately, a trial with this parameter gave the worst fit (largest condition number and largest standard deviations). A possible explanation is the very small value of the Cartesian coordinate of $\mathrm{N}$ : $b=0.030 \AA$ (semiexperimental value). For this reason, the bond length $\mathrm{C} \cdots \mathrm{O}$ was used as parameter to be fitted. The number of parameters to be fitted has to be determined. The analysis of the rotational spectra and the small value of the ground-state inertial defect $\left(0.050 \mathrm{u} \AA^{2}\right)$ showed that the complex is planar, as later confirmed by the ab initio calculations. Furthermore, the ab initio calculations do not indicate any significant deviation from linearity of the NNO group. Thus, six parameters are enough to determine the structure. The results of the fit are given in Table VII. The geometrical parameters of the moieties $\mathrm{N}_{2} \mathrm{O}$ and $\mathrm{CO}$ are accurate. On the other hand, the $r(\mathrm{C} \cdots \mathrm{O})$ bond length is short: $r_{\mathrm{e}}=3.291(5) \AA$ but with a large uncertainty. Nevertheless, this result is in satisfactory 
agreement with the $r_{\mathrm{e}}(\mathrm{IV})$ ab initio structures. It is interesting to compare this result with what is obtained when the bond lengths of the monomers are fixed: $r_{\mathrm{e}}=3.2921(2) \AA$. Obviously, in this latter case, the uncertainty is much too small and it demonstrates the superiority of the mixed regression method. Actually, in spite of the predicates, the fit with the mixed regression is not well conditioned. Furthermore, although the rovibrational corrections have probably the correct order of magnitude, they are obviously not accurate enough, which is not surprising taking into account the results of sections 1.2 and 3.2. In particular, the residuals for the $B$-rotational constants are large. A typical example of the inaccuracy of the rovibrational corrections is the value of the $r(\mathrm{C}=\mathrm{O})$ bond length calculated with the help of Kraitchman's equations. ${ }^{13}$ It mainly depends on the two largest Cartesian coordinates: the $a$-coordinate of the oxygen atom and the $a$-coordinate of the carbon atom (the $b$ coordinates are quite small) which are proportional to the square root of $\Delta I_{b}$. As these coordinates are large, the derived bond length should be accurate. Unfortunately, it is far from true. Using the computed rovibrational corrections $\left(\Delta B\left({ }^{13} \mathrm{C}^{16} \mathrm{O}\right)=47.854 \mathrm{MHz}, \Delta B\left({ }^{13} \mathrm{C}^{18} \mathrm{O}\right)=44.514 \mathrm{MHz}\right)$ gives $r_{\mathrm{e}}$ $=1.0855 \AA$ to be compared to the expected value $1.1285 \AA$. It is enough to multiply the two $\Delta B$ by $1.0236\left(\Delta B\left({ }^{13} \mathrm{C}^{16} \mathrm{O}\right)=48.984 \mathrm{MHz}, \Delta B\left({ }^{13} \mathrm{C}^{18} \mathrm{O}\right)=45.565 \mathrm{MHz}\right)$ to obtain the correct result see Table VIII. Actually, simulations show that it is enough to modify the rovibrational corrections of the $B$ rotational constants by a few hundred $\mathrm{kHz}$ to obtain a structure in good agreement with the best ab initio calculations. In particular, an attempt to optimize the $\Delta B$ rovibrational corrections by the method of predicate observations using as predicates the three bond lengths $\mathrm{C}=\mathrm{O}, \mathrm{N}=\mathrm{N}$, and $\mathrm{N}=\mathrm{O}$ with an uncertainty of $0.001 \AA$ and the computed $\Delta B$ with an uncertainty of $2 \%$ permitted to obtain satisfactory results, see Table S-IX of the Supplementary Material. This confirms the sensitivity of the structure to small errors in the rovibrational corrections.

\subsection{Mass-dependent structure}

The conclusion is that the anharmonic force field is perhaps not reliable enough and cannot be used to calculate accurately the small variations of the rovibrational corrections upon isotopic substitution. Fortunately, there is an alternative way to obtain a near- equilibrium structure using the massdependent $\left(r_{\mathrm{m}}\right)$ method, based on the expansion of the ground state moments of inertia $I_{g}^{0}$ to

$$
I_{g}^{0}=I_{g}^{m}+c_{g} \sqrt{I_{g}^{m}}+d_{g}\left[\frac{\prod m_{i}}{M}\right]^{1 /(2 n-2)}
$$

where $I_{g}^{m}$ is the moment of inertia calculated from the $r_{m}$ parameters (principal inertial axes: $g=a$, $b, c), c_{g}$ and $d_{g}$ are fitting parameters (one for each axis), $M$ is the total molecular mass, $m_{i}$ are the atomic masses and $n$ is the number of atoms. When the last term is neglected, it results in the socalled $r_{m}^{(1)}$ structure. The correction term (with the coefficient $c_{g}$ ) essentially scales the moment of 
inertia with the correct mass dependence. When the second correction term is retained (with the coefficient $d_{g}$ ), it gives the $r_{m}^{(2)}$ structure. ${ }^{74}$ This second term may be important in the presence of small coordinates. If the parameters $c_{g}$ and $d_{g}$ are both fixed at zero, it gives simply the effective structure or $r_{0}$. The weak point of this method is that it requires the determination of three to six additional parameters, which may considerably worsen the fit and, for that reason, is rarely successful for a moderately large molecule. However, it was shown in the case of ethynylcyclohexane ${ }^{75}$ that the method gives a structure whose quality is comparable to that of the semiexperimental method. This conclusion was strengthened on several other molecules including fructose ${ }^{76}$ and diallyldisulfide. ${ }^{77}$ Furthermore, Kisiel tested the applicability of the $r_{\mathrm{m}}$ method to molecular complexes and obtained satisfactory results. ${ }^{78}$ The same predicate values as for the semiexperimental fit (previous section) were used. The results of the fit are also given in Table VII. The agreement with the best ab initio structure is not satisfactory for the intermolecular bond length which seems to be much too long. It confirms that Eqn. (3) is only a rough approximation.

\section{CONCLUSION}

It is now almost routine work to determine the structure of a rigid molecule with an accuracy of a few milliangström. However, the present work shows that it is difficult to obtain intermolecular bond lengths with an accuracy better than a tenth of an $\AA$ for intermolecular clusters. This conclusion is true both for ab initio calculations and experimental determinations. On the other hand, it seems that intermolecular bond angles can still be determined with an accuracy of a few tenths of a degree, provided the $\operatorname{CSSD}(\mathrm{T})$ method is employed. On the other hand, the MP2 method fails to calculate accurate bond angles as is often the case when the bending force constant is small. More generally, the $\operatorname{CCSD}(\mathrm{T})$ method gives satisfactory results provided a very large basis set is used including diffuse functions and taking into account the counterpoise correction. The semiexperimental method also gives satisfactory results although the accuracy of the intermolecular bond length $\mathrm{C} \cdots \mathrm{O}$ is lower than for the other parameters. On the other hand, in this particular case, the mass-dependent method disappoints.

In Table VII, the structures determined in this work are listed. Excluding the $\operatorname{CCSD}(\mathrm{T}) \_$ae/wCVQZ (because the basis set is obviously too small) and the $r_{m}^{(2)}$ structure which gives a much too large $\mathrm{r}\left(\mathrm{C}^{\cdots} \mathrm{O}\right)$ bond length $(3.338(4) \AA$ instead of a median value of 3.300(9) $\mathrm{A})$, there are twelve different structures of the complex. One way to obtain a better estimate is to calculate their median because it is a robust estimator. It gives for the intermolecular parameters: $r(\mathrm{C} \cdots \mathrm{O})=$ $3.300(9) \AA ̊ ․$ $9.40(30)^{\circ}$. For these values, the standard deviation (in parentheses) is derived from the median 
absolute deviation (MAD) of the residuals ( $s=\mathrm{MAD} / 0.6745)$. Comparison of these median values with the results of Table VII shows that the best results are obtained with the CP-CCSD(T)/AV6Z level of theory, $r\left(\mathrm{IV}_{6}\right)$, nicely confirming the conclusion of section 1.3.4. Although the extrapolation methods do not significantly improve the accuracy, it is worth noting that the "cheap" extrapolation $\mathrm{CP}-\mathrm{CCSD}(\mathrm{T}) / \mathrm{AV} \infty \mathrm{Z}(\mathrm{T} \rightarrow \mathrm{Q})$ gives results close to those given by the median with $r(\mathrm{C} \cdots \mathrm{O})=3.296$ $\AA ; R_{\mathrm{cm}}=3.818 \AA ; \angle \mathrm{CO} 2 \mathrm{~N} 2=74.91^{\circ} ; \angle \mathrm{OCO}=145.98^{\circ}$. Finally, it appears that the semiexperimental method is less accurate, in particular for the angles: $\angle \mathrm{CO} 2 \mathrm{~N} 2$ at $74.6^{\circ}$ is $0.5^{\circ}$ too small and $\angle \mathrm{OCO}$ at $147.0^{\circ}$ is $1^{\circ}$ too large. It is interesting to compare these results with those found by Qian and Howard ${ }^{16}$ who determined the three following parameters $R_{\mathrm{cm}}=3.8707(3) \AA, \theta_{1}=80 \pm 5^{\circ}$ and $\theta_{2}=$ $20 \pm 5^{\circ}$ with the aid of an intermolecular potential calculation.

Although the van der Waals bond was shown to be between the carbon atom of $\mathrm{CO}$ and the oxygen atom of $\mathrm{N}_{2} \mathrm{O}$, it is worth noting that the sum of the van der Waals radii $r(\mathrm{C})+r(\mathrm{~N})=1.77+$ $1.66=3.43 \AA$ is much larger than the distance $\mathrm{C}^{\cdots} \mathrm{N} 2,3.22 \AA$ whereas $r(\mathrm{C})+r(\mathrm{O})=1.77+1.50=$ $3.27 \AA$ which is of the same order of magnitude as the bond distance $\mathrm{C}^{\cdots} \mathrm{O} 2,3.30 \AA$ (the van der Waals bond radii are taken from Ref. ${ }^{79}$ ).

The geometrical structure of the monomers is hardly affected by the complexation. Furthermore, the redistribution of charges is almost negligible. Thus, it should be possible to estimate the electric dipole moment of the dimer by a simple vectorial addition. Using $\mu\left(\mathrm{N}_{2} \mathrm{O}\right)=0.1608 \mathrm{D},{ }^{80}$ $\mu(\mathrm{CO})=0.1098 \mathrm{D},{ }^{81}$ and $\theta_{3}=109.7^{\circ}$ (Table VII) gives $\mu\left(\mathrm{N}_{2} \mathrm{O} \cdots \mathrm{CO}\right)=0.16 \mathrm{D}$. This value is smaller than the CP-B2PLYP-D3/AVQZ result of $0.29 \mathrm{D}$. A CCSD(T)/AVQZ calculation gives a smaller value, $0.20 \mathrm{D}$, in better agreement with the vector addition. Nevertheless, is known that the experimental dipole moment of a dimer is larger than the value calculated from vector addition of free monomers. The difference is due to the neglect of the polarizabilities of the monomers. ${ }^{82}$

The present work offers future prospects for the investigation of molecular structures of intermolecular complexes, advancing the collaboration between computational and experimental methods.

\section{SUPPLEMENTARY MATERIAL}

Figure S-I and Tables S-I to S-VIII containing additional computational and experimental data.

\section{ACKNOWLEDGEMENTS}

This work was supported by the Dr. B. Mez-Starck Foundation (Germany). Y.J., M.J., R.T.S. and A.L. acknowledge funding from the MICINN-FEDER (grant PGC2018-098561-B-C22). 


\section{DATA AVAILABILITY STATEMENT}

The data that supports the findings of this study are available within the article and its supplementary material. 


\section{REFERENCES}

${ }^{1}$ A.M. Maharramov, K.T. Mahmudov, M.N. Kopylovich, and A.J.L. Pombeiro, editors, NonCovalent Interactions in the Synthesis and Design of New Compounds (John Wiley \& Sons, Inc, Hoboken, NJ, 2016).

${ }^{2}$ J.J. Novoa, editor, Intermolecular Interactions in Crystals (The Royal Society of Chemistry, 2018).

${ }^{3}$ G. Gilli and P. Gilli, The Nature of the Hydrogen Bond (Oxford University Press, Oxford, 2009).

${ }^{4}$ S. Scheiner, editor, Noncovalent Forces (Springer International Publishing, Cham, Switzerland, 2015).

${ }^{5}$ I. Alkorta, J. Elguero, and A. Frontera, Crystals 10, (2020).

${ }^{6}$ J.-P. Schermann, Spectroscopy and Modeling of Biomolecular Building Blocks (Elsevier, 2008).

${ }^{7}$ P. Hobza and K. Muller-Dethlefs, Non-Covalent Interactions (Royal Society of Chemistry, Cambridge, 2009).

${ }^{8}$ P. Kraus, D.A. Obenchain, and I. Frank, J. Phys. Chem. A 122, 1077 (2018).

${ }^{9}$ K.L. Bak, J. Gauss, P. Jørgensen, J. Olsen, T. Helgaker, and J.F. Stanton, J. Chem. Phys. 114, 6548 (2001).

${ }^{10}$ W. Caminati and J.-U. Grabow, in Front. Adv. Mol. Spectrosc., edited by J. Laane (Elsevier Inc., 2018), pp. 569-598.

${ }^{11}$ W. Caminati and J.-U. Grabow, in Front. Mol. Spectrosc., edited by J. Laane (Elsevier, Amsterdam, 2009), pp. 455-552.

${ }^{12}$ M. Juanes, R.T. Saragi, W. Caminati, and A. Lesarri, Chem. - A Eur. J. 25, 11402 (2019).

${ }^{13}$ J. Kraitchman, Am. J. Phys. 21, 17 (1953).

${ }^{14}$ J. Demaison, J.E. Boggs, and A.G. Csaszar, editors, Equilibrium Molecular Structures (CRC Press, Boca Raton, Florida, 2016).

${ }^{15}$ Z. Kisiel, E. Białkowska-Jaworska, and L. Pszczółkowski, J. Chem. Phys. 109, 10263 (1998).

${ }^{16}$ J. Řezáč and P. Hobza, Chem. Rev. 116, 5038 (2016).

${ }^{17}$ L. Goerigk, A. Hansen, C. Bauer, S. Ehrlich, A. Najibi, and S. Grimme, Phys. Chem. Chem. Phys. 19, 32184 (2017).

${ }^{18}$ Y. Xu and A.R.W. McKellar, J. Mol. Spectrosc. 180, 164 (1996).

${ }^{19}$ H.-B. Qian and B.J. Howard, J. Mol. Spectrosc. 184, 156 (1997).

${ }^{20}$ M.S. Ngarî, Y. Xu, and W. Jäger, J. Mol. Spectrosc. 197, 244 (1999).

${ }^{21}$ L.C. Krisher and L. Pierce, J. Chem. Phys. 32, 1619 (1960).

${ }^{22}$ M. Venayagamoorthy and T.A. Ford, J. Mol. Struct. THEOCHEM 717, 111 (2005).

${ }^{23}$ A.J. Barclay, C. Lauzin, S. Sheybani-Deloui, K.H. Michaelian, and N. Moazzen-Ahmadi, Phys. Chem. Chem. Phys. 19, 1610 (2017).

${ }^{24}$ C. Møller and M.S. Plesset, Phys. Rev. 46, 618 (1934).

${ }^{25}$ G.D. Purvis and R.J. Bartlett, J. Chem. Phys. 76, 1910 (1982).

${ }^{26}$ K. Raghavachari, G.W. Trucks, J.A. Pople, and M. Head-Gordon, Chem. Phys. Lett. 157, 479 (1989).

${ }^{27}$ W. Kohn and L.J. Sham, Phys. Rev. 140, A1133 (1965).

${ }^{28}$ C. Lee, W. Yang, and R.G. Parr, Phys. Rev. B 37, 785 (1988).

${ }^{29}$ A.D. Becke, J. Chem. Phys. 98, 5648 (1993).

${ }^{30}$ S. Grimme, J. Chem. Phys. 124, 034108 (2006).

${ }^{31}$ S. Grimme, J. Comput. Chem. 27, 1787 (2006).

${ }^{32}$ S. Grimme, S. Ehrlich, and L. Goerigk, J. Comput. Chem. 32, 1456 (2011).

${ }^{33}$ T.H. Dunning, J. Chem. Phys. 90, 1007 (1989).

${ }^{34}$ R.A. Kendall, T.H. Dunning, and R.J. Harrison, J. Chem. Phys. 96, 6796 (1992).

${ }^{35}$ J.M.L. Martin, Chem. Phys. Lett. 242, 343 (1995).

${ }^{36}$ K.A. Peterson and T.H. Dunning, J. Chem. Phys. 117, 10548 (2002).

${ }^{37}$ L. Margulès, J. Demaison, and H.D. Rudolph, J. Mol. Struct. 599, 23 (2001).

${ }^{38}$ E. Papajak and D.G. Truhlar, J. Chem. Theory Comput. 6, 597 (2010). 
${ }^{39}$ M.J. Frisch, J.A. Pople, and J.S. Binkley, J. Chem. Phys. 80, 3265 (1984).

${ }^{40}$ S.F. Boys and F. Bernardi, Mol. Phys. 19, 553 (1970).

${ }^{41}$ R.F.W. Bader, Atoms in Molecules, A Quantum Theory (Clarendon Press, 1994).

42 R.J. Gillespie and P.L.A. Popelier, Chemical Bonding and Molecular Geometry (Oxford University Press, Oxford, 2001).

${ }^{43}$ J. Cioslowski and S.T. Mixon, J. Am. Chem. Soc. 113, 4142 (1991).

${ }^{44}$ J. Cioslowski, Chem. Phys. Lett. 194, 73 (1992).

45 J. Cioslowski and P.R. Surján, J. Mol. Struct. THEOCHEM 255, 9 (1992).

${ }^{46}$ J. Cioslowski, A. Nanayakkara, and M. Challacombe, Chem. Phys. Lett. 203, 137 (1993).

${ }^{47}$ J. Cioslowski and A. Nanayakkara, Chem. Phys. Lett. 219, 151 (1994).

${ }^{48}$ J. Cioslowski and B.B. Stefanov, Mol. Phys. 84, 707 (1995).

${ }^{49}$ B.B. Stefanov and J. Cioslowski, J. Comput. Chem. 16, 1394 (1995).

${ }^{50}$ H.-J. Werner, P.J. Knowles, G. Knizia, F.R. Manby, and M. Schütz, WIREs Comput Mol Sci 2, 242 (2012).

${ }^{51}$ H.-J. Werner, P.J. Knowles, G. Knizia, F.R. Manby, M. Schütz, others, P. Celani, W. Györffy, D. Kats, T. Korona, R. Lindh, A. Mitrushenkov, G. Rauhut, K.R. Shamasundar, T.B. Adler, R.D. Amos, S.J. Bennie, A. Bernhardsson, A. Berning, D.L. Cooper, M.J.O. Deegan, A.J. Dobbyn, F. Eckert, E. Goll, C. Hampel, A. Hesselmann, G. Hetzer, T. Hrenar, G. Jansen, C. Köppl, S.J.R. Lee, Y. Liu, A.W. Lloyd, Q. Ma, R.A. Mata, A.J. May, S.J. McNicholas, W. Meyer, T.F. Miller III, M.E. Mura, A. Nicklass, D.P. O’Neill, P. Palmieri, D. Peng, K. Pflüger, R. Pitzer, M. Reiher, T. Shiozaki, H. Stoll, A.J. Stone, R. Tarroni, T. Thorsteinsson, M. Wang, and M. Welborn, (2019).

${ }^{52}$ M.J. Frisch, G.W. Trucks, H.B. Schlegel, G.E. Scuseria, M.A. Robb, J.R. Cheeseman, G.

Scalmani, V. Barone, G.A. Petersson, H. Nakatsuji, X. Li, M. Caricato, A. V. Marenich, J. Bloino, B.G. Janesko, R. Gomperts, B. Mennucci, H.P. Hratchian, J. V. Ortiz, A.F. Izmaylov, J.L.

Sonnenberg, Williams, F. Ding, F. Lipparini, F. Egidi, J. Goings, B. Peng, A. Petrone, T. Henderson, D. Ranasinghe, V.G. Zakrzewski, J. Gao, N. Rega, G. Zheng, W. Liang, M. Hada, M. Ehara, K. Toyota, R. Fukuda, J. Hasegawa, M. Ishida, T. Nakajima, Y. Honda, O. Kitao, H. Nakai, T. Vreven, K. Throssell, J.A. Montgomery Jr., J.E. Peralta, F. Ogliaro, M.J. Bearpark, J.J. Heyd, E.N. Brothers, K.N. Kudin, V.N. Staroverov, T.A. Keith, R. Kobayashi, J. Normand, K.

Raghavachari, A.P. Rendell, J.C. Burant, S.S. Iyengar, J. Tomasi, M. Cossi, J.M. Millam, M. Klene, C. Adamo, R. Cammi, J.W. Ochterski, R.L. Martin, K. Morokuma, O. Farkas, J.B. Foresman, and D.J. Fox, (2016).

${ }^{53}$ T.J. Balle and W.H. Flygare, Rev. Sci. Instrum. 52, 33 (1981).

${ }^{54}$ U. Andresen, H. Dreizler, J.-U. Grabow, and W. Stahl, Rev. Sci. Instrum. 61, 3694 (1990).

${ }^{55}$ J.-U. Grabow, W. Stahl, and H. Dreizler, Rev. Sci. Instrum. 67, 4072 (1996).

${ }^{56}$ A.E. Reed, R.B. Weinstock, and F. Weinhold, J. Chem. Phys. 83, 735 (1985).

${ }^{57}$ A. V. Marenich, S. V. Jerome, C.J. Cramer, and D.G. Truhlar, J. Chem. Theory Comput. 8, 527 (2012).

${ }^{58}$ D.A. Obenchain, L. Spada, S. Alessandrini, S. Rampino, S. Herbers, N. Tasinato, M.

Mendolicchio, P. Kraus, J. Gauss, C. Puzzarini, J.-U. Grabow, and V. Barone, Angew. Chemie Int. Ed. 57, 15822 (2018).

${ }^{59}$ S. Grimme, J. Comput. Chem. 25, 1463 (2004).

${ }^{60}$ P. Kraus and I. Frank, J. Phys. Chem. A 122, 4894 (2018).

${ }^{61}$ L. Spada, N. Tasinato, F. Vazart, V. Barone, W. Caminati, and C. Puzzarini, Chem. - A Eur. J. 23, 4876 (2017).

${ }^{62}$ C. Puzzarini, J.F. Stanton, and J. Gauss, Int. Rev. Phys. Chem. 29, 273 (2010).

${ }^{63}$ C. Puzzarini, J. Phys. Chem. A 113, 14530 (2009).

${ }^{64}$ S. Coriani, D. Marchesan, J. Gauss, C. Hättig, T. Helgaker, and P. Jørgensen, J. Chem. Phys. 123, 184107 (2005).

${ }^{65}$ J.-L. Teffo and A. Chédin, J. Mol. Spectrosc. 135, 389 (1989).

${ }^{66}$ J. Demaison and N. Vogt, Accurate Structure Determination of Free Molecules (Lectures Notes in Chemistry, Vol. 105) (Springer International Publishing, Cham, 2020). 
${ }^{67}$ M. Juanes, N. Vogt, J. Demaison, I. León, A. Lesarri, and H.D. Rudolph, Phys. Chem. Chem. Phys. 19, 29162 (2017).

${ }^{68}$ T.H. Dunning, J. Phys. Chem. A 104, 9062 (2000).

${ }^{69}$ L.S. Bartell, D.J. Romanesko, and T.C. Wong, in Chem. Soc. Spec. Period. Rep. - No. 20, edited by G.A. Sim and L.E. Sutton (London, 1975), p. 72.

${ }^{70}$ A. Belsey, Conditioning Diagnostics: Collinearity and Weak Data in Regression (Wiley, New York, NY, 1991).

${ }^{71}$ J. Demaison, in Equilib. Mol. Struct., edited by J. Demaison, J.E. Boggs, and A.G. Csaszar (CRC Press, Boca Raton, Florida, 2016), pp. 29-52.

${ }^{72}$ W. Gordy and R.L. Cook, Microwave Molecular Spectra (John Wiley \& Sons, Inc, New York, NY, 1984).

${ }^{73}$ T.A. Ruden, T. Helgaker, P. Jørgensen, and J. Olsen, J. Chem. Phys. 121, 5874 (2004).

${ }^{74}$ J.K.G. Watson, A. Roytburg, and W. Ulrich, J. Mol. Spectrosc. 196, 102 (1999).

${ }^{75}$ N. Vogt, J. Demaison, H.D. Rudolph, M. Juanes, J. Fernández, and A. Lesarri, J. Chem. Phys. 148, 1 (2018).

${ }^{76}$ N. Vogt, J. Demaison, E.J. Cocinero, P. Écija, A. Lesarri, H.D. Rudolph, and J. Vogt, Phys. Chem. Chem. Phys. 18, 15555 (2016).

${ }^{77}$ J. Demaison, N. Vogt, R.T. Saragi, M. Juanes, H.D. Rudolph, and A. Lesarri, Phys. Chem. Chem. Phys. 21, 19732 (2019).

78 Z. Kisiel, J. Mol. Spectrosc. 218, 58 (2003).

${ }^{79}$ S. Alvarez, Dalt. Trans. 42, 8617 (2013).

${ }^{80}$ J.M.L.J. Reinartz, W.L. Meerts, and A. Dymanus, Chem. Phys. 31, 19 (1978).

${ }^{81}$ J.S. Muenter, J. Mol. Spectrosc. 55, 490 (1975).

${ }^{82}$ S.J. Harris, S.E. Novick, W. Klemperer, and W.E. Falconer, J. Chem. Phys. 61, 193 (1974). 
FIG. 1. The two isomers of $\mathrm{N}_{2} \mathrm{O} \cdots \mathrm{CO}$, showing the principal inertial axes system. The isomer corresponding to global minimum on the potential energy surface (with a $\mathrm{C} \cdots \mathrm{O}$ interaction, see text) is shown in the upper row.

See Attached file "Figure-1.tif" 
FIG. 2. Typical rotational transitions of the ${ }^{15} \mathrm{~N}_{2} \mathrm{O} \cdots{ }^{12} \mathrm{CO}$ and ${ }^{15} \mathrm{~N}_{2} \mathrm{O} \cdots{ }^{13} \mathrm{CO}$ isotopologues, recorded with a Balle-Flygare pulsed-jet microwave spectrometer (quantum numbers $J_{K_{-1}, K_{+1}}$ ). The resonance frequencies correspond to the average of the two observed Doppler components (in red).

See Attached file "Figure-2.jpg" 
TABLE I. AIM analysis of $\mathrm{N}_{2} \mathrm{O} \cdots \mathrm{CO}$ performed at the B3LYP/6-311+G(3df) level of theory.

\begin{tabular}{|c|c|c|c|c|c|c|c|c|}
\hline & \multicolumn{4}{|c|}{ complex } & \multicolumn{4}{|c|}{ monomers } \\
\hline & $r / \AA$ & $\rho$ & $\nabla$ & $\varepsilon$ & $r / \AA$ & $\rho$ & $\nabla$ & $\varepsilon$ \\
\hline $\mathrm{CO} 1$ & 1.1240 & $5.1810^{-1}$ & 0.233 & $2.4310^{-4}$ & 1.1245 & 0.52 & 0.268 & $1.3310^{-2}$ \\
\hline $\mathrm{C}^{\cdots} \mathrm{N} 2$ & 3.4650 & & & & & & & \\
\hline $\mathrm{NN}$ & 1.1208 & $5.9610^{-1}$ & -1.795 & $7.6810^{-4}$ & 1.1208 & 0.6 & -1.795 & $1.9110^{-14}$ \\
\hline $\mathrm{NO} 2$ & 1.1823 & $5.4110^{-1}$ & -1.173 & $3.0310^{-4}$ & 1.1823 & 0.54 & -1.170 & $8.2210^{-15}$ \\
\hline $\mathrm{C}^{\cdots} \mathrm{O} 2$ & 3.5057 & $3.6810^{-3}$ & 0.013 & $6.4010^{-1}$ & & & & \\
\hline$\angle \mathrm{OCN} 2$ & 163.96 & & & & & & & \\
\hline
\end{tabular}


TABLE II. Atomic charges on $\mathrm{N}_{2} \mathrm{O} \cdots \mathrm{CO}(\mathrm{au})$.

\begin{tabular}{lcccc}
\hline Atom & \multicolumn{2}{c}{$q$ (complex) } & \multicolumn{2}{c}{$q$ (monomer) } \\
& $\mathrm{NBO}$ & $\mathrm{CM} 5$ & $\mathrm{NBO}$ & $\mathrm{CM} 5$ \\
\hline $\mathrm{O}$ & -0.484 & -0.125 & -0.490 & -0.125 \\
$\mathrm{C}$ & 0.484 & 0.125 & 0.490 & 0.125 \\
$\mathrm{~N} 2$ & 0.424 & 0.165 & 0.421 & 0.165 \\
$\mathrm{~N} 1$ & -0.084 & -0.087 & -0.085 & -0.087 \\
$\mathrm{O}$ & -0.339 & -0.077 & -0.336 & -0.077 \\
\hline
\end{tabular}


Table III. Bond length $r\left(\mathrm{C}^{\cdots} \mathrm{O}\right)(\AA)$, dipole moment $\mu(\mathrm{D})$, centrifugal distortion constants $D_{J}, D_{J K}$, $D_{K}, d_{l}$ and $d_{2}(\mathrm{kHz})$, inertial defect $\Delta\left(\mathrm{u}^{2}\right)$, rovibrational corrections $\left(A_{\mathrm{e}}-A_{0}\right)$, etc. $(\mathrm{MHz})$ and vibrational frequencies $\left(\mathrm{cm}^{-1}\right)$ for $\mathrm{N}_{2} \mathrm{O} \cdots \mathrm{CO}$.

\begin{tabular}{|c|c|c|c|c|c|c|c|c|c|}
\hline$\overline{\text { Method }^{a}}$ & \multirow[t]{2}{*}{$\exp ^{\mathrm{a}}$} & & \multicolumn{3}{|c|}{ CP-B2PLYP-D3 b } & \multicolumn{3}{|c|}{ CP-B3LYP-D3 $^{b}$} & \multirow{2}{*}{$\begin{array}{l}\text { CP-MP2 } \\
\text { 6-311+G* }\end{array}$} \\
\hline Basis set & & \multicolumn{2}{|c|}{ mAVQZ } & \multicolumn{2}{|c|}{$6-311+G(3 d f)$} & AVTZ & \multicolumn{2}{|c|}{ AVTZ } & \\
\hline$r(\mathrm{C} \cdots \mathrm{O})$ & & 3.3444 & & 3.338198 & & 3.3343 & 3.313514 & & \\
\hline$\mu$ & & 0.2584 & & 0.2846 & & 0.2506 & 0.19202 & & \\
\hline$D_{J}$ & $8.2214(25)$ & 5.9278 & & 6.1272 & & 5.806 & 5.7871 & & \\
\hline$D_{J K}$ & $359.551(17)$ & 373.7944 & & 375.5446 & & 378.825 & 410.2017 & & \\
\hline$D_{K}$ & {$[-298.08]$} & -298.0763 & & -286.9446 & & -298.820 & -211.5483 & & \\
\hline$d_{1}$ & $-1.3142(18)$ & -0.9042 & & -0.9255 & & -0.893 & 0.8339 & & \\
\hline$d_{2}$ & $-0.6585(26)$ & -0.5570 & & -0.5570 & & -0.570 & -0.5852 & & \\
\hline$\Delta$ & 1.935 & 1.975 & & 1.982 & & & 1.822 & & \\
\hline$A_{\mathrm{e}}-A_{0}$ & & -111.916 & & -111.034 & & & -124.089 & & \\
\hline$B_{\mathrm{e}}-B_{0}$ & & 47.377 & & 48.576 & & & 44.221 & & \\
\hline \multirow[t]{2}{*}{$C_{\mathrm{e}}-C_{0}$} & & 44.319 & & 45.284 & & & 41.925 & & \\
\hline & & $\mathrm{E}(\text { harm })^{\mathrm{b}}$ & $(\text { anharm })^{b}$ & $\mathrm{E}(\text { harm })^{\mathrm{b}}$ & $\mathrm{E}($ anharm $)$ & $\mathrm{E}(\text { harm })^{b}$ & $E(\text { harm })^{b}$ & $\mathrm{E}(\text { anharm })^{\mathrm{b}}$ & $\mathrm{E}(\text { harm })^{b}$ \\
\hline as. $\mathrm{N}_{2} \mathrm{O}$ stretch & $2226.662^{c}$ & 2269.8 & 2212.0 & 2271.6 & 2212.9 & 2263.7 & 2345.1 & 2288.5 & 2246.4 \\
\hline CO stretch & $2147.1823^{d}$ & 2167.3 & 2139.0 & 2171.1 & 2145.3 & 2158.4 & 2213.2 & 2187.6 & 2127.8 \\
\hline sym. $\mathrm{N}_{2} \mathrm{O}$ stretch & & 1307.4 & 1297.8 & 1309.6 & 1300.1 & 1300.7 & 1326.0 & 1314.7 & 1288.1 \\
\hline$\gamma\left(\mathrm{N}_{2} \mathrm{O}\right)$ oop & & 601.6 & 598.3 & 602.0 & 596.5 & 599.7 & 613.8 & 610.1 & 537.7 \\
\hline$\delta\left(\mathrm{N}_{2} \mathrm{O}\right)$ ip & & 598.3 & 591.2 & 599.1 & 592.1 & 596.5 & 617.5 & 610.2 & 537.3 \\
\hline libration ip & & 98.7 & 122.7 & 98.8 & 74.1 & 99.3 & 102.7 & 117.2 & 99.2 \\
\hline ip vibration & & 61.9 & 8.6 & 61.5 & 49.1 & 62.8 & 65.0 & 22.0 & 65.5 \\
\hline CO libration oop & & 44.4 & 57.5 & 45.0 & 39.7 & 44.2 & 44.1 & 44.9 & 48.5 \\
\hline $\mathrm{C} \cdots \mathrm{O}$ stretch & $33^{\mathrm{a}}$ & 24.4 & 78.7 & 24.2 & 65.8 & 24.2 & 23.9 & 70.1 & 29.1 \\
\hline
\end{tabular}

a This work unless otherwise stated.

${ }^{\mathrm{b}} \mathrm{CP}=$ counterpoise correction applied, (harm) - harmonic approximation, (anharm) - anharmonic approximation.

${ }^{\mathrm{c}}$ Ref. $\left({ }^{19}\right)$.

${ }^{\mathrm{d}}$ Ref. $\left({ }^{18}\right)$. 
TABLE IV. Core correlation effects (for distances in $\AA$ and angles in degrees).

\begin{tabular}{|c|c|c|c|c|c|c|c|c|c|c|}
\hline & \multirow{2}{*}{\multicolumn{2}{|c|}{$\begin{array}{c}\text { MP2 } \\
\text { wCVTZ } \\
\text { ae }\end{array}$}} & \multirow[b]{2}{*}{$\mathrm{fc}-\mathrm{ae}$} & \multicolumn{7}{|c|}{$\operatorname{CCSD}(\mathrm{T})$} \\
\hline & & & & $\begin{array}{c}\text { wCVTZ } \\
\text { fc }\end{array}$ & $\begin{array}{c}\text { wCVTZ } \\
\text { ae }\end{array}$ & $\mathrm{fc}-\mathrm{ae}$ & $\begin{array}{c}\text { wCVQZ } \\
\text { fc }\end{array}$ & $\begin{array}{c}\text { wCVQZ } \\
\text { ae }\end{array}$ & $\begin{array}{c}\text { wCVQZ } \\
\text { fc }- \text { ae }\end{array}$ & $\begin{array}{c}\mathrm{wCV} \propto \mathrm{Z}^{\mathrm{a}} \\
\mathrm{fc}-\mathrm{ae}\end{array}$ \\
\hline$\overline{\mathrm{CO} 1}$ & 1.1357 & 1.1336 & 0.0021 & 1.1329 & 1.1309 & 0.0020 & 1.1305 & 1.1282 & 0.0023 & 0.0025 \\
\hline $\mathrm{CN} 2$ & 3.1997 & 3.1939 & 0.0058 & 3.2473 & 3.2435 & 0.0037 & 3.2405 & 3.2368 & 0.0037 & 0.0036 \\
\hline O1CN2 & 174.671 & 174.635 & 0.036 & 165.955 & 166.17 & -0.22 & 166.682 & 166.790 & -0.11 & -0.03 \\
\hline $\mathrm{NN}$ & 1.1525 & 1.1500 & 0.0025 & 1.1304 & 1.1285 & 0.0018 & 1.1283 & 1.1262 & 0.0021 & 0.0023 \\
\hline $\mathrm{CNN}$ & 93.499 & 93.476 & 0.024 & 96.381 & 96.31 & 0.07 & 96.021 & 95.931 & 0.09 & 0.11 \\
\hline $\mathrm{NO} 2$ & 1.1782 & 1.1766 & 0.0016 & 1.1883 & 1.1865 & 0.0018 & 1.1867 & 1.1847 & 0.0020 & 0.0022 \\
\hline $\mathrm{CN} 2 \mathrm{O} 2$ & 86.418 & 86.438 & -0.019 & 83.544 & 83.62 & -0.07 & 83.912 & 84.010 & -0.10 & -0.11 \\
\hline $\mathrm{C}^{\cdots} \mathrm{O} 2$ & 3.3400 & 3.3345 & 0.0055 & 3.3300 & 3.3276 & 0.0024 & 3.3307 & 3.3287 & 0.0020 & 0.0017 \\
\hline $\mathrm{CO} 2 \mathrm{~N} 2$ & 72.967 & 72.942 & 0.026 & 75.689 & 75.628 & 0.06 & 75.338 & 75.260 & 0.08 & 0.09 \\
\hline $\mathrm{O} 2 \mathrm{CN} 2$ & 20.614 & 20.621 & -0.007 & 20.767 & 20.753 & 0.01 & 20.750 & 20.730 & 0.02 & 0.03 \\
\hline $\mathrm{O} 1 \mathrm{CO} 2$ & 154.06 & 154.01 & 0.043 & 145.19 & 145.42 & -0.23 & 145.93 & 146.06 & -0.13 & -0.05 \\
\hline
\end{tabular}

${ }^{\mathrm{a}}$ Extrapolation, see Eq. (1). 
TABLE V. Contribution of the diffuse functions calculated at the MP2 level (for distances in $\AA$ and angles in degrees).

\begin{tabular}{lcccc}
\hline & AVTZ & AVQZ & AV5Z & AV6Z \\
\hline $\mathrm{CO} 1$ & 0.0003 & 0.0005 & 0.0002 & 0.0002 \\
$\mathrm{CN} 2$ & -0.0607 & -0.0328 & -0.0085 & -0.0083 \\
$\mathrm{O} 1 \mathrm{CN} 2$ & 2.04 & 0.17 & -0.05 & 0.07 \\
$\mathrm{NN}$ & 0.0003 & 0.0003 & 0.0002 & 0.0001 \\
$\mathrm{CNN}$ & -0.80 & -0.05 & 0.08 & 0.01 \\
$\mathrm{NO} 2$ & 0.0010 & 0.0006 & 0.0002 & 0.0001 \\
$\mathrm{CN} 2 \mathrm{O} 2$ & 0.72 & -0.01 & -0.10 & -0.03 \\
$\mathrm{C} \cdots \mathrm{O} 2$ & -0.0423 & -0.0307 & -0.0097 & -0.0084 \\
$\mathrm{CO} 2 \mathrm{~N} 2$ & -1.03 & -0.20 & 0.03 & -0.02 \\
$\mathrm{O} 2 \mathrm{CN} 2$ & 0.31 & 0.21 & 0.07 & 0.06 \\
$\mathrm{O} 1 \mathrm{CO} 2$ & 1.73 & -0.04 & -0.12 & 0.01 \\
\hline
\end{tabular}


Table VI. Counterpoise correction calculated at the M2 level of theory (distances in $\AA$ and angles in degrees).

\begin{tabular}{lllllllll}
\hline & & $\mathrm{CO} 1$ & $\mathrm{C}^{\cdots} \mathrm{N} 2$ & $\mathrm{C}^{\cdots} \mathrm{O} 2$ & $\mathrm{NN}$ & $\mathrm{NO} 2$ & $\mathrm{O} 1 \mathrm{CO} 2$ & $\mathrm{CN} 2 \mathrm{~N}$ \\
\hline AVQZ & $\mathrm{CP}^{\mathrm{a}}$ & 1.1347 & 3.1821 & 3.3298 & 1.1513 & 1.1781 & 154.41 & 93.14 \\
& no $^{\mathrm{b}}$ & 1.1347 & 3.1578 & 3.3064 & 1.1513 & 1.1780 & 154.36 & 93.15 \\
& $\mathrm{BSSE}^{\mathrm{c}}$ & 0.0000 & 0.0243 & 0.0234 & 0.0000 & 0.0001 & 0.05 & -0.01 \\
$\mathrm{AV5Z}$ & $\mathrm{CP}^{\mathrm{a}}$ & 1.1339 & 3.1732 & 3.3207 & 1.1505 & 1.1775 & 154.32 & 93.16 \\
& no $^{\mathrm{b}}$ & 1.1339 & 3.1624 & 3.3105 & 1.1505 & 1.1774 & 154.35 & 93.16 \\
& $\mathrm{BSSE}^{\mathrm{c}}$ & 0.0000 & 0.0108 & 0.0102 & 0.0000 & 0.0001 & -0.03 & -0.01 \\
$\mathrm{AV6Z}$ & $\mathrm{CP}^{\mathrm{a}}$ & 1.1337 & 3.1691 & 3.3155 & 1.1501 & 1.1772 & 154.20 & 93.21 \\
& no $^{\mathrm{b}}$ & 1.1336 & 3.1620 & 3.3101 & 1.1501 & 1.1772 & 154.33 & 93.14 \\
& $\mathrm{BSSE}^{\mathrm{c}}$ & 0.0000 & 0.0072 & 0.0059 & 0.0000 & 0.0000 & -0.14 & 0.07 \\
$\mathrm{AV} \infty \mathrm{Z}$ & $\mathrm{CP}^{\mathrm{a}}$ & 1.1333 & 3.1636 & 3.3083 & 1.1497 & 1.1770 & 154.02 & 93.29 \\
& $\mathrm{no}^{\mathrm{b}}$ & 1.1333 & 3.1613 & 3.3095 & 1.1497 & 1.1770 & 154.31 & 93.11 \\
& $\mathrm{BSSE}^{\mathrm{c}}$ & 0.0000 & 0.0023 & -0.0012 & 0.0000 & 0.0000 & -0.28 & 0.18 \\
\hline
\end{tabular}

${ }^{\mathrm{a}} \mathrm{CP}=$ with counterpoise correction.

${ }^{\mathrm{b}}$ No counterpose correction.

${ }^{\mathrm{c}}$ Basis set superposition error. 
Table VII. Equilibrium structures of the $\mathrm{N}_{2} \mathrm{O} \cdots \mathrm{CO}$ complex and its monomers (distances in $\AA$ and angles in degrees) ${ }^{\mathrm{a}}$.

\begin{tabular}{|c|c|c|c|c|c|c|c|c|c|c|c|c|}
\hline & Method & Basis set & $r(\mathrm{~N}=\mathrm{N})$ & $r(\mathrm{~N}=\mathrm{O})$ & $r\left(\mathrm{C}^{\cdots} \mathrm{O}\right)$ & $\angle \mathrm{CO} 2 \mathrm{~N} 2$ & $r(\mathrm{C}=\mathrm{O})$ & $\angle \mathrm{OCO}$ & $R_{\mathrm{cm}}^{\mathrm{b}}$ & $\theta_{1}{ }^{\mathrm{c}}$ & $\theta_{2}{ }^{\mathrm{c}}$ & $\theta_{3}{ }^{\mathrm{c}}$ \\
\hline & $\begin{array}{l}\text { monomers } \\
\text { CCSD(T)_ae } \\
\text { complex }\end{array}$ & $\operatorname{CCSD}(\mathrm{T}) \_\mathrm{ae}$ & wCVQZ & 1.1851 & & & 1.1282 & & & & & \\
\hline$r(\mathrm{Q})$ & $\operatorname{CCSD}(\mathrm{T}) \_\mathrm{ae}$ & wCVQZ & 1.1262 & 1.1847 & 3.3287 & 75.260 & 1.1282 & 146.06 & 3.857 & 80.2 & 9.4 & 109.1 \\
\hline$r(5)$ & & wCV5Z & 1.1254 & 1.1845 & 3.3117 & 75.048 & 1.1278 & 146.18 & 3.837 & 80.4 & 9.2 & 108.8 \\
\hline$r(6)$ & & wCV6Z & 1.1252 & 1.1843 & 3.3100 & 75.094 & 1.1276 & 146.03 & 3.836 & 81.3 & 10.3 & 109.0 \\
\hline$r(\infty)$ & & $\mathrm{wCV} \infty \mathrm{Z}$ & 1.1249 & 1.1841 & 3.3077 & 75.156 & 1.1274 & 145.83 & 3.834 & 80.2 & 9.4 & 109.2 \\
\hline$r(\mathrm{II})$ & & $\mathrm{AV} \infty \mathrm{Z}$ & 1.1250 & 1.1843 & 3.3063 & 75.109 & 1.1275 & 146.03 & 3.833 & 80.3 & 9.3 & 109.0 \\
\hline$r(\mathrm{III})$ & $\operatorname{CCSD}(\mathrm{T})$ & $\mathrm{V} \infty \mathrm{Z}$ & 1.1249 & 1.1846 & 3.2942 & 74.830 & 1.1275 & 146.23 & 3.816 & 80.5 & 9.0 & 108.5 \\
\hline$r\left(\left(\mathrm{IV}_{\mathrm{Q}}\right)\right.$ & CP-CCSD(T) & AVQZ & 1.1266 & 1.1858 & 3.3145 & 75.06 & 1.1290 & 145.63 & 3.840 & 80.2 & 9.4 & 109.3 \\
\hline$r\left(\left(\mathrm{IV}_{5}\right)\right.$ & & AV5Z & 1.1257 & 1.1849 & 3.3025 & 75.17 & 1.1281 & 145.60 & 3.830 & 80.1 & 9.6 & 109.5 \\
\hline \multirow[t]{4}{*}{$r\left(\left(\mathrm{IV}_{6}\right)\right.$} & & AV6Z & 1.1254 & 1.1847 & 3.2973 & 75.21 & 1.1278 & 145.47 & 3.825 & 80.0 & 9.7 & 109.6 \\
\hline & & $\mathrm{AV} \infty \mathrm{Z}(\mathrm{T} \rightarrow \mathrm{Q})$ & 1.1240 & 1.1834 & 3.2956 & 74.91 & 1.1260 & 145.98 & 3.818 & 80.4 & 9.2 & 108.8 \\
\hline & & $\mathrm{AV} \infty \mathrm{Z}(\mathrm{Q} \rightarrow 5)$ & 1.1248 & 1.1840 & 3.2954 & 75.17 & 1.1271 & 145.37 & 3.820 & 80.0 & 9.7 & 109.7 \\
\hline & & $\mathrm{AV} \infty \mathrm{Z}(5 \rightarrow 6)$ & 1.1249 & 1.1844 & 3.2901 & 75.27 & 1.1275 & 145.30 & 3.819 & 79.9 & 9.8 & 109.8 \\
\hline \multicolumn{3}{|c|}{ Diatomic approximation } & & & & & & & 3.811 & 83.8 & 9.4 & \\
\hline \multirow{2}{*}{\multicolumn{2}{|c|}{$\begin{array}{l}r_{\mathrm{e}}^{S E} \\
r_{\mathrm{m}}^{(2)}\end{array}$}} & & $1.1260(16)$ & $1.1856(12)$ & $3.291(5)$ & $74.6(2)$ & $1.1267(27)$ & $147.0(3)$ & 3.811 & 81.9 & 9.4 & 107.6 \\
\hline & & & $1.1263(12)$ & $1.1846(17)$ & $3.338(4)$ & $74.42(8)$ & $1.1258(20)$ & $144.01(5)$ & 3.843 & 81.7 & 12.1 & 110.4 \\
\hline
\end{tabular}

${ }^{a}$ See Table S-III for the details.

${ }^{b}$ Distance between the centers of masses of $\mathrm{CO}$ and $\mathrm{N}_{2} \mathrm{O}\left(\mathrm{cm}_{1}\right.$ and $\mathrm{cm}_{2}$, respectively).

${ }^{\mathrm{c}} \theta_{1}=\angle\left(\mathrm{cm}_{1} \ldots \mathrm{cm}_{2} \ldots \mathrm{O} 2\right), \theta_{2}=\angle\left(\mathrm{cm}_{2} \ldots \mathrm{cm}_{1} \ldots \mathrm{C}\right), \theta_{3}$ is an angle between the OC and $\mathrm{N}_{2} \mathrm{O}$ axes, see Fig. 1 for definitions. 
TABLE VIII. Equilibrium coordinates of the $\mathrm{C}=\mathrm{O}$ moiety derived from Kraitchman's equations and rovibrational corrections $\Delta B$.

\begin{tabular}{lrrrr}
\hline & ${ }^{12} \mathrm{C}^{16} \mathrm{O}^{\mathrm{a}}$ & ${ }^{13} \mathrm{C}^{18} \mathrm{O}$ & ${ }^{12} \mathrm{C}^{16} \mathrm{O}$ & $r(\mathrm{CO}) / \AA$ \\
\hline Original & & & & \\
$\Delta B / \mathrm{MHz}$ & 47.854 & 44.514 & 45.553 & 1.0855 \\
$a / \AA$ & & 2.7711 & 1.7043 & \\
$b / \AA$ & & 0.0407 & -0.1600 & \\
Scaled & & & & \\
$\Delta B / \mathrm{MHz}$ & 48.954 & 45.565 & 45.553 & 1.1285 \\
$a / \AA$ & & 2.7705 & 1.6600 & \\
$b / \AA$ & & 0.0407 & -0.1601 & \\
\hline
\end{tabular}

${ }^{a}$ Parent species.

${ }^{b}$ See text. 

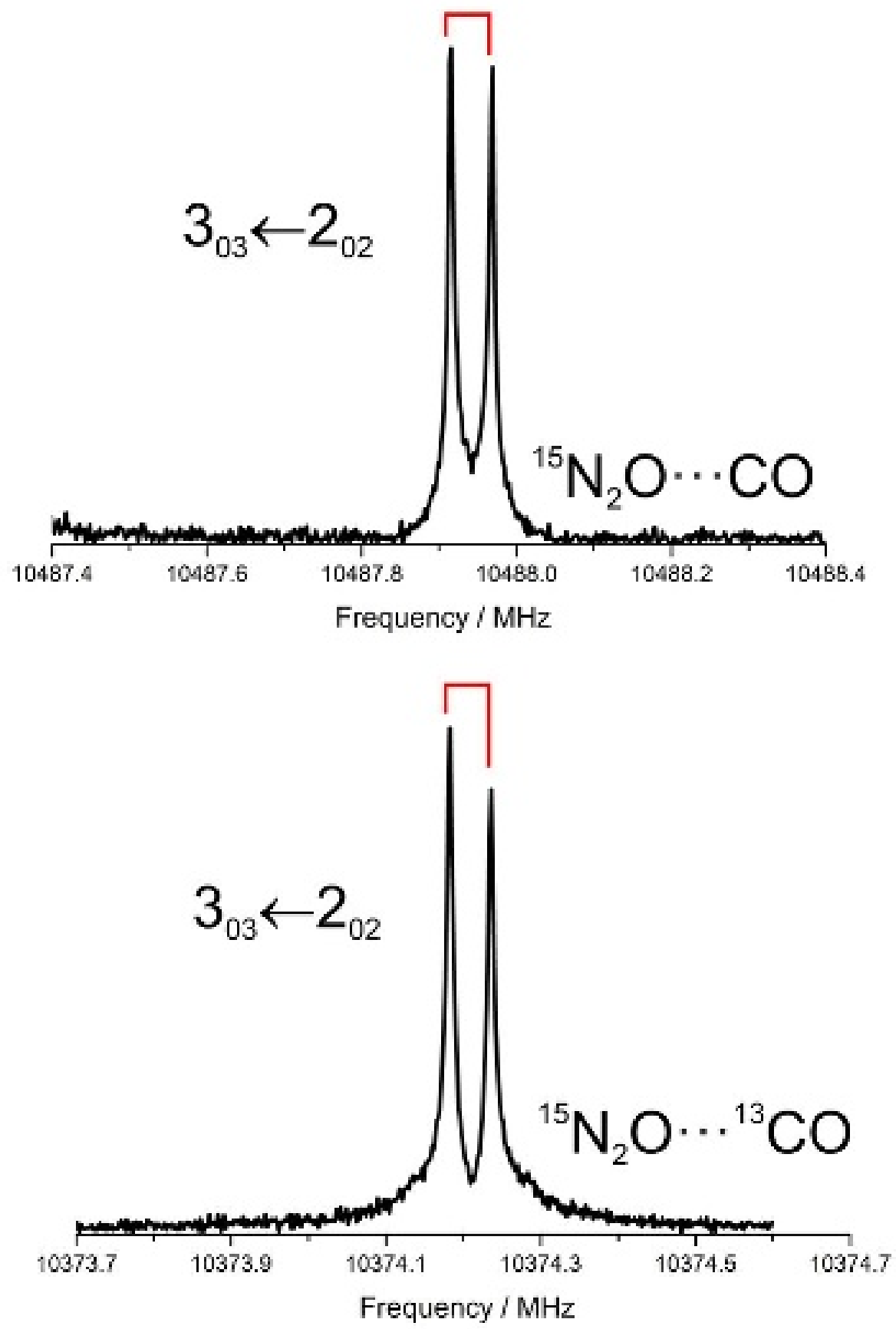